\title{
Droplet Trajectories of Wave Impact Sea Spray on a Marine Vessel
}

\author{
S.R. Dehghani ${ }^{1}$, Y.S. Muzychka, G.F. Naterer \\ Department of Mechanical Engineering, Faculty of Engineering and Applied Science, \\ Memorial University of Newfoundland, St. John’s, NL, A1B 3X5, Canada
}

\section{Abstract}

Marine icing phenomena are strongly dependent on the rate of water impact on marine vessels. The most important component is wave-impact sea spray. There is limited understanding of droplet size and velocity distributions of wave-impact sea spray. Initial distributions of size and velocity of droplets are crucial for the calculation of the droplet path and consequently for determining the water impact on surfaces. This paper develops a new model of wave-impact sea spray by using a distribution of the size and velocity of droplets at the edge of the vessel. The concepts of water-sheet breakup and droplet breakup lead to an inverse dependence between the size and velocity of droplets after the breakup process. Droplets take different paths and form a spray cloud in front of the vessel. The liquid water content in front of the vessel can be calculated by considering the arrangement, sizes, and velocities of a set of droplets in the spray cloud. The response of the droplet trajectory model to various initial conditions with different sets of droplet sizes and velocities is examined. The numerical results are compared to real data from field observations. Droplet sizes are inversely proportional to droplet velocities, as verified by liquid water content data obtained by the field observations. This paper proposes the use of this inverse relationship based on physics of the breakup process, as the initial data for calculating the wave-impact sea spray trajectory in front of a vessel.

Keywords: Marine Icing, Wave Impact, Sea Spray, Droplet Trajectory, Sheet Breakup, Droplet Breakup

\footnotetext{
${ }^{1}$ Corresponding author, Postdoctoral Fellow, Phone: +1 (709) 771-6216, Email: srdehghani@mun.ca
} 


\section{1. Introduction}

25 Marine icing phenomena, involving ice accretion and accumulation on vessels, have been significant challenges in offshore technology development. Due to growing expectations about the role of the Arctic

27 in supplying oil and natural gas resources, the importance of marine icing phenomena has increased.

28 Accurate prediction of ice accretion on marine vessels has remained a challenging concern. Researchers

29 have made many efforts in this field, but several aspects of these phenomena are not yet well understood (Kulyakhtin and Tsarau, 2014; Shipilova et al., 2012; Ryerson, 2011).

Atmospheric and sea-generated droplets are two sources of water droplet delivery. Field observations show that atmospheric droplets and humidity, including rain, snow, drizzle, and fog, are not the most significant contributors to ice accretion, and consequently, they are not the main potential causes for ice accretion on marine objects in harsh conditions. These have been analysed in detail by Makkonen (1984). On the other hand, sea-generated water droplets have more significant roles in the occurrence of ice creation and accumulation on marine objects (Lozowski et al., 2000; Zakrzewski, 1987). Wind-generated spray, which refers to droplets raised from the sea surface by wind, and wave-impactgenerated spray, caused by atomized droplets created by wave impact on the outer surface of marine objects, are two important sources of sea-generated droplets. Wind-generated droplets generally have lesser effects on marine icing phenomena, and they are often neglected in the calculations of ice accretion (Lozowski et al., 2000; Zakrzewski, 1987). Wave-impact-generated droplets are the main cause of ice accretion on marine objects. In harsh conditions at sea, marine vessels are faced with a high rate of water impact due to the differing impacts of high-energy waves (Zakrzewski, 1987). Figure 1 shows a general schematic of the sources of the water droplets delivered to marine vessels and their role in marine icing modeling. 


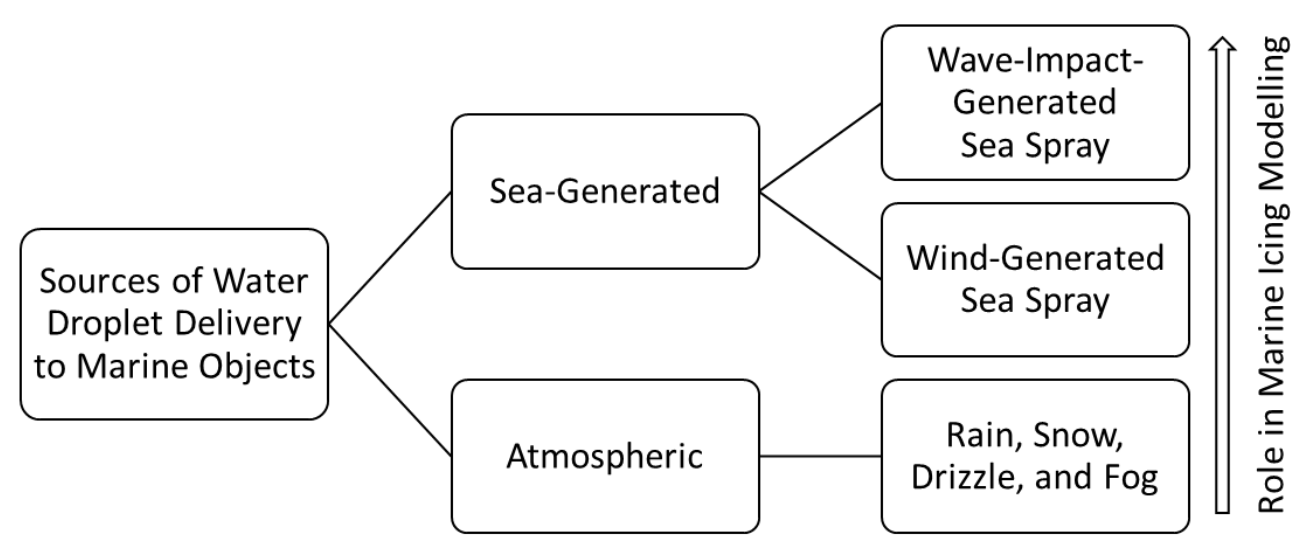

Fig. 1. Sources of water impact and their role in marine icing modelling

(1)

.

列

.

A review of previous studies shows that there are limited field observations or the measurement of the rate of water delivery to marine objects due to wave impact. Some correlations and empiricallybased relations have been developed. These relations describe the rates of incoming water of a waveimpact sea spray, which are usually the amounts of water in a unit volume of air, called the Liquid Water Content (LWC). The most significant investigations of wave-impact sea spray are from Borisenkov et al. (1975), Zakrzewski (1987), Horjen and Carstens (1989), and Ryerson (1995). These attempted to extract LWC data from field observations. The vertical distributions for liquid water content have been the most useful data obtained from the field observations. These LWC relations have been used by researchers to predict ice accretion on vessels.

The main focus of this paper is on wave-impact sea spray in front of vessels. There have been a few past works which are directly related to vessels. Borisenkov et al. (1975) showed that the LWC, which results from wave-impact splashes, varies by height from deck level exponentially. The correlation and model were based on observations in the Sea of Japan obtained by a Medium-sized Fishing Vessel (MFV). It reported the amount of incoming water to the deck without reporting the size and velocity of droplets.

The report of Borisenkov et al. (1975) was the first and most significant investigation which is related to vertical distribution of LWC in front of a vessel. 
Zakrzewski (1987) extended the work of Borisenkov et al. (1975) by generalizing the correlation

67 for all wave conditions. It was assumed that the LWC depends on wave height and that the relative velocity of a wave and vessel can increase the LWC by a power of one and two respectively. The generalized relation was verified by Borisenkov's correlation. Ryerson (1995) included aspects of droplet size distribution and droplet concentration for wave-impact sea sprays. The observations showed that there

71 is a range of droplet diameters from very fine, at $14 \mu \mathrm{m}$, to very large, at 7,700 $\mu \mathrm{m}$. In addition, it was

72 reported that the mean droplet concentration was $4 \times 10^{5} \mathrm{drops} / \mathrm{m}^{3}$. This gives more information than 73 the liquid water content alone.

Calculations and predictions of ice accretion on a surface are strongly dependent on the rate of water droplet impact at every location of that surface on marine vessels. The rate of water droplet delivery depends on droplet trajectories from the injection spots, which are the upper edges of the vessel bow, to 77 target surfaces. The gravity force, wind velocity, initial size of droplets, initial velocity of droplets, and many other parameters can affect droplet trajectories and, consequently, the rate of water received on target surfaces (Zakrzewski and Lozowski, 1988). The crucial data for applying a droplet trajectory analysis 80 are droplet size distribution, droplet velocity distribution, and droplet concentration (Dehghani et al., 81 2009). With this information, the geometry of the vessels or offshore structures, and some atmospheric 82 information, a good estimation of sea water droplet delivery to the surface points can be predicted. 83 Therefore, without a sophisticated model for the distribution of the droplet sizes and velocities, it is difficult to accurately predict the rate of droplet water impingement on target surfaces; as a result, the estimate of the amount of accumulated ice will not be accurate. Past studies have reported relations that explain the vertical distribution of LWC due to the impact 87 of waves on vessels and offshore superstructures (Forest et. al, 2005; Zakrzewski, 1987; Lozowski et al., 88 2000). These involve functions of wave specifications, atmospheric situations and marine object 89 characteristics. Estimating the accumulated ice needs the contribution of the droplet trajectory method 
to give a good estimation of water impact on the surface. Past studies have typically assumed droplet

91 sizes, velocities, and concentrations in order to calculate the droplet trajectories and, consequently, the

92 ice accretion results. Lozowski et al. (2000) used a RIGICE model and assumed the initial vertical and

93 horizontal velocities and initial drop sizes. Other researchers who used the droplet trajectory have not

94 used distributed droplet sizes and droplet velocities. They usually assumed a fixed initial velocity and size

95 for all heights (Shipilova et al., 2012; Horjen, 2013; Kulyakhtin and Tsarau, 2014).

The complete set of data of wave-impact sea spray should include the distribution of droplet size,

97 velocity, and concentration. To date, there has been no past work to describe a wave-impact sea spray by

98 considering this essential information. In addition, phenomena related to the creation of the wave-impact

99 sea spray have not yet been explained (Zakrzewski, 1987; Lozowski et al., 2000; Shipilova et al., 2012;

100 Horjen, 2013; Kulyakhtin and Tsarau, 2014).

101

Ryerson (1995) reported the size and concentration of droplets. This was based on the measured

samples from some regions of a spray cloud and not a complete distribution data set for the whole spray

cloud. The user would need to generalize the suggested distribution for the droplet size and concentration

104 to all regions of the spray cloud. In addition, having an initial velocity distribution for droplets is crucial to

obtain the path of a spray cloud on the vessel and Ryerson's model did not include the droplet velocity

106 data.

There have been previous investigations into determining the LWC for offshore structures. In those cases, the geometry of the surfaces that waves impact is different from the vessel's bow. Their wave-impact sea spray would be different. Forest et al. (2005) reviewed past LWC relations for offshore structures. The exponential form of those relations is the same as the relations for the vessels but they 111 have some significant differences. As mentioned, the most relevant formula for the vertical distribution 112 of LWC for vessels is a model of Borisenkov et al. (1975), and developed by Zakrzewski (1987). The formula 113 presented by Borisenkov et al. (1975) is one of the formulae based on real field observations in the Sea of 
Japan by using the MFV. It is at least valid for that special situation, that vessel geometry, and the

115 environmental situation.

In the present work, a new correlation between droplet sizes and velocities at the injection area,

117 the top edge of the bow, is introduced. This size-velocity dependence for droplets aids in the use of the

118 LWC information. The new model will be examined in comparison with the LWC from field observations

119 for real vessels (Borisenkov et al. 1975), and a sensitivity analysis will show the effect of various

120 parameters on the output of the model.

121

122 2. Wave-Impact Sea Spray

123 Dividing the marine icing phenomena into two consequent phenomena, called off-deck and on-deck

124 phenomena in this study, will clarify the importance of wave-impact sea spray. The off-deck phenomena

125 are those that occur outside of the marine vessel. These include sea wave creation and movement, sea

126 wave impact on marine objects, water breakup, and finally droplet dispersion in front of the marine

127 object. This set of distributed water droplets in front of a vessel is called wave-impact sea spray. On the

128 other hand, the on-deck phenomena are those which occur on and above the marine objects. The spray

129 and droplet trajectory, droplet impingement to marine objects, start of icing, and ice accretion are the

130 main subdivisions of the on-deck phenomena.

131 The majority of past research has been focused on on-deck phenomena. There have been many

132 reports on ice accretion, which is the final stage of on-deck phenomena. But there is a limited number of

133 studies about the impact of sea water droplets on vessels. It should be noted that the quality and accuracy

134 of the prediction of ice accretion is strongly dependent on the rate of incoming water droplets on marine

135 vessels. The estimation of off-deck phenomena is a prerequisite for accurate prediction of on-deck

136 phenomena. But off-deck phenomena are lesser-known parts of the marine icing phenomena. Figure 2

137 illustrates the classification of marine icing phenomena based on the above-mentioned description. 


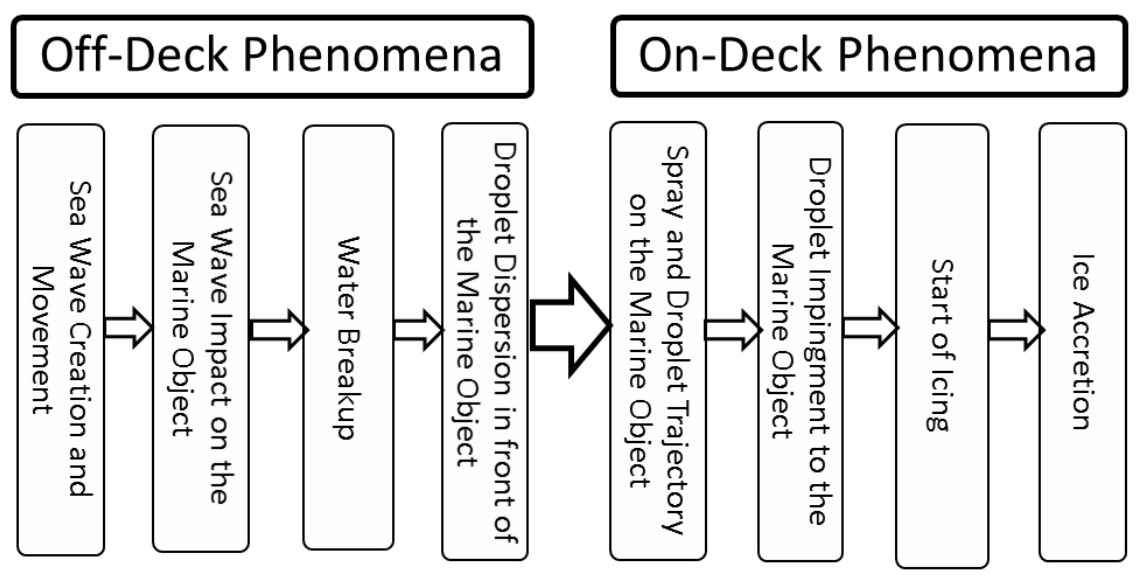

A review of past research related to wave-impact sea spray for a vessel's bow shows that this

143 field of research was initially pursued with field observation data (Borisenkov et al., 1975). There are

144 variations of LWC for various heights, vessel speeds, heading angles, wind speeds, wave specifications,

145 sea water temperatures, wind temperatures and some other information related to the icing situation.

146 These were examined by Zakrzewski (1987) and Lozowski et al. (2000) by generalizing a specific correlation

147 to various situations. Information about LWC is not sufficient for estimating the rate of water impact on

148 every point of a vessel. Size, velocity and concentration of the droplets are the other elements that are

149 required for the droplet trajectory. There have been some attempts to find a good estimation of size and

150 velocity of droplets in a cloud of wave-impact sea spray. However, none can yield a distribution of size

151 and velocity of the droplets in front of a vessel. Some of these attempts assumed the velocity of droplets

152 to be the same as the wind velocity. In MARICE, the initial velocity, which is related to the wave

153 specification and heading angle, was proposed as a model (Lozowski et al., 2000; Shipilova et al., 2012;

154 Horjen, 2013; Kulyakhtin and Tsarau, 2014). None of the previous works used a distribution of droplet size

155 as initial sizes for droplet trajectories (Zakrzewski, 1987; Lozowski et al., 2000; Shipilova et al., 2012; 
Horjen, 2013; Kulyakhtin and Tsarau, 2014). Therefore it is important to have a good estimation of the

157 distribution of droplet size and velocity to be able to use the droplet trajectory method and calculate the 158 rate of water delivery to every point of a vessel.

Droplet sizes and velocities are a result of the breakup of sea water. Evaluating this breakup mechanism leads to a relation between droplet sizes and velocities. In the present paper, the vessel bow 161 is chosen as a sample of an inclined surface that has the potential for conducting water-sheet breakup.

162 The other points of the vessel which are in direct contact with seawater have a similar potential for 163 creating a wave-impact sea spray. Impacting waves on those regions can cause water breakup and droplet 164 creation which will create the cloud of wave-impact sea spray.

The wave impact on a vessel bow is the starting step of sea-spray cloud formation. Similar to other 166 impacts of water on vertical or inclined rigid surfaces, an upward thin water sheet is expected to form on 167 the bow. The local impact velocity of the water particles on the bow is one of the main criteria for the 168 quality of the thin water sheet creation. It determines the velocity and thickness of the water sheet. This 169 sheet of water can slip on the bow or separate from the bow at an angle. In case of low-velocity impact 170 and the consequent low velocity of sheets, the sheet water may stay unified and continue its movement. 171 High-velocity impacts can create a high-velocity water sheet leading to surface breakup and, 172 consequently, to droplet breakup. High-velocity sheets cannot keep unity, and they break into many small 173 parts. Long water strips are one result of sheet breakup and water droplets are the final result of the 174 secondary breakup. The most important stages of wave-impact sea spray phenomena are shown in Fig. 3. 175 


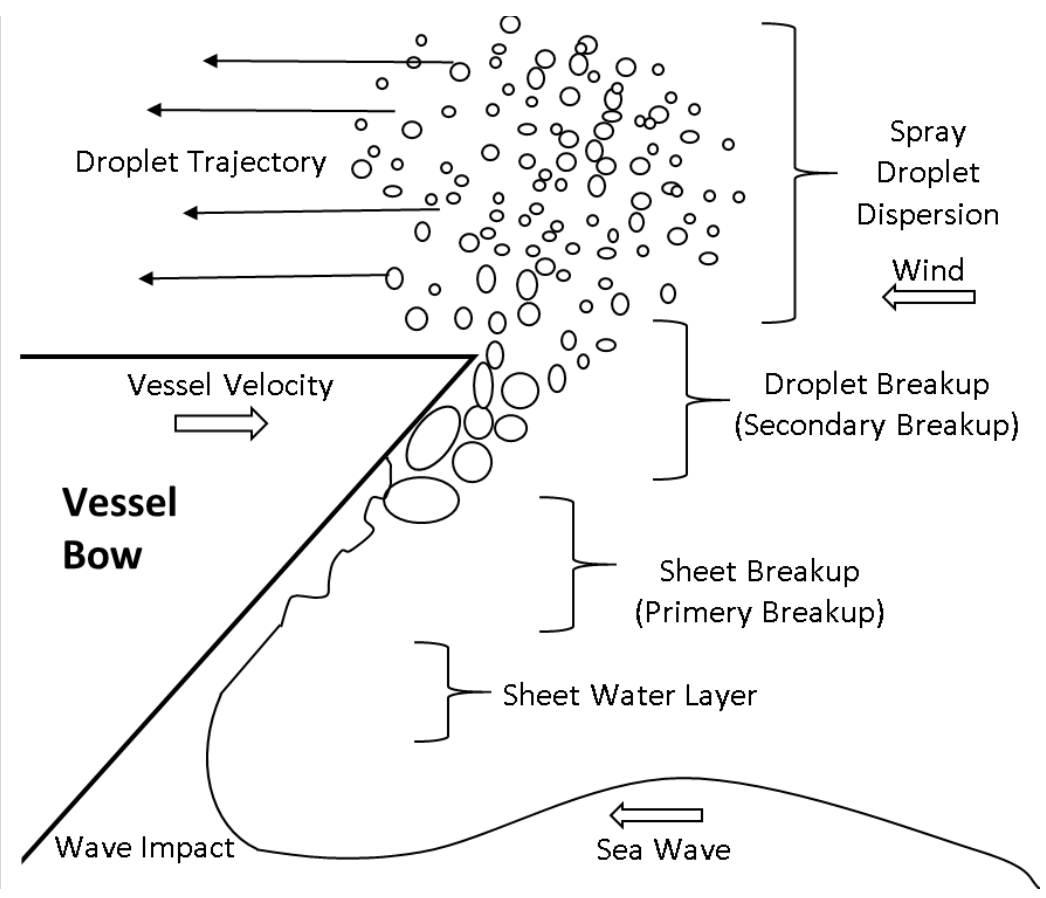

Fig. 3. Illustration of the wave impact, water sheet creation, and breakup stages

The stages are, in order: wave impact on the bow, water sheet creation on the bow, sheet breakup, droplet breakup, and droplet dispersion in front of the vessel. The last stage is known as a sea spray cloud in front of the vessel. The wind velocity, vessel velocity, and the other contributors to this process are shown in Fig. 3.

The impact of a single sea wave on a bow occurs at various velocities. Impact velocity is the relative velocity of water particles with respect to the bow in the contact area. Due to the variation of contact areas, and different local velocities of water particles of the wave, the impact velocity will be variable. It is worth considering that, in the short period of the impact, the velocity of the impact varies from a minimum effective velocity to a maximum supplied velocity. The minimum effective velocity is the minimum velocity that is required for the creation of a sheet of water. The maximum supplied velocity is the maximum velocity of impact that is possible to occur between the wave and vessel. This behaviour of velocity variation makes spray-cloud modelling more complex. 
In some cases, the impact velocity is sufficient to create a sheet of water in effective periods of

192 impact. The impact velocity must be high to to create a thin sheet of water on the bow. In the short 193 effective period of impact, the bow experiences a range of impact velocities that make various sheets of 194 water on the bow. A sheet of water is formed on the bow because the water particles of the wave have 195 enough energy to overcome gravity. Sometimes a sheet of water is formed, but the breakup does not 196 occur. This implies that there are more criteria involved in the breakup phenomenon (Ahmed et al., 2009). Breakup occurs when two portions of fluid or a single portion of fluid forms a free surface with surface energy. A system which is not at the minimum level of energy will attempt to rearrange and move toward the lower energy level, leading to the breakup of the fluid into smaller portions to minimize the system surface energy by reducing the surface area. The exact outcome of the breakup process is 201 dependent on the surface tension, viscosity, density, and diameter of the thread undergoing breakup. In 202 other words, breakup will occur when inertia forces, which are representatives of density, velocity and 203 diameter, can be controlled by surface tension forces. In this case, existing instabilities can grow and create water strips and droplets (Sazhin 2014). Some of the sheet conditions on the bow may be able to 205 satisfy the criteria of water breakup and atomization. In this case, the breakup starts to split the water 206 sheet into smaller parts. High-velocity air flow, in the presence of the liquid sheet water on the bow, can create significant 208 shear development at the interface between the two fluids. The shear gives rise to a Kelvin-Helmholtz 209 type instability, causing the sheet to be disturbed, and as the oscillating amplitude grows, the sheet will 210 split into divided parts. Stretching causes the sheet to tear into ligaments. The breakup mechanisms 211 appear to be independent of the water viscosity and surface tension; however, these properties affect 212 the final drop size distribution (Lozano et al. 1998). As mentioned, the impact velocity is not constant. Therefore, the sheet velocity and the resultant 214 droplet velocities are not constant (Sarchami et al. 2010). In every single impact, the bow is faced with a 
range of impact velocities that create various sheet velocities and therefore, various sizes of droplets.

216 Low-velocity impacts make big droplets with a low velocity; these droplets are stable and cannot be

217 further divided. The high-velocity droplets are unstable and split into smaller droplets. They continue their

218 division to reach a stable condition, involving a balance between the inertia and surface tension at the

219 end of the breakup process. The sizes of the droplets depend on their velocities: higher velocities lead

220 droplets to resize to finer droplets, while the medium velocity droplets may split up into medium-sized

221 droplets. Therefore, at the end of droplet breakup there are various sizes of droplets with different

222 velocities: the bigger droplets have low velocities and the smaller droplets have high velocities (Sazhin

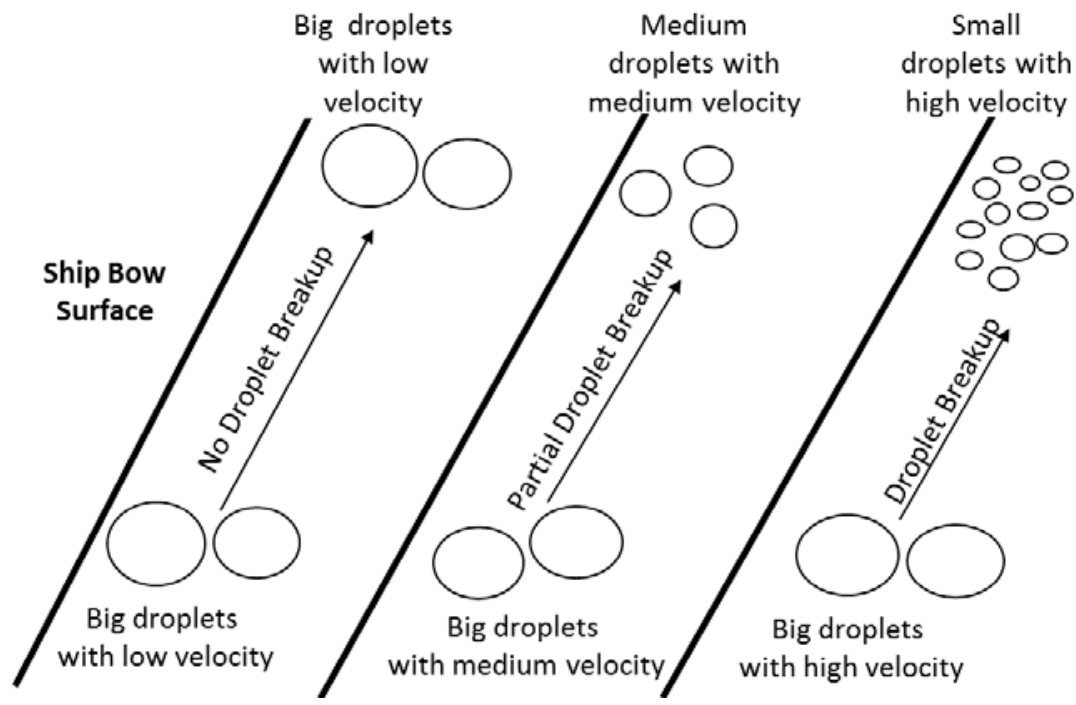

Fig. 4. Three types of droplet breakup mechanisms and size-velocity dependence

After the breakup stage, the droplets continue their paths in front of the bow. At this time, the wave-impact sea spray is visible and a spray cloud appears in front of the vessel. The forces exerted on 
232 them to change their direction and travel in the direction of the wind and gravity. Aspects of this

233 procedure can be calculated by using the droplet trajectory method in front of the vessel.

In past research, sea spray creation due to bow-wave interaction has not been well understood.

235 The main interest of studying bow-wave interaction has been the calculation of the force exerted on the

236 bow or the amount of direct water on the deck (Gu et al. 2014; Greco and Lugni, 2012; Greco et al., 2012;

237 Kapsenberg, 2011). There is a similar phenomenon that can be used for understanding these stages. Sheet

238 breakup on a rigid surface has been investigated in splash plates by several researchers. A splash plate

239 creates splash and spray droplets by using the impact of water on an inclined surface plate (Ahmed et al.

240 2009; Bussmann et al., 2000; Sarchami et al., 2010; Ashgriz et al., 1996). This procedure includes sheet

241 water breakup and droplet breakup, which are fundamentally very similar to the breakup mechanisms on

242 the bow.

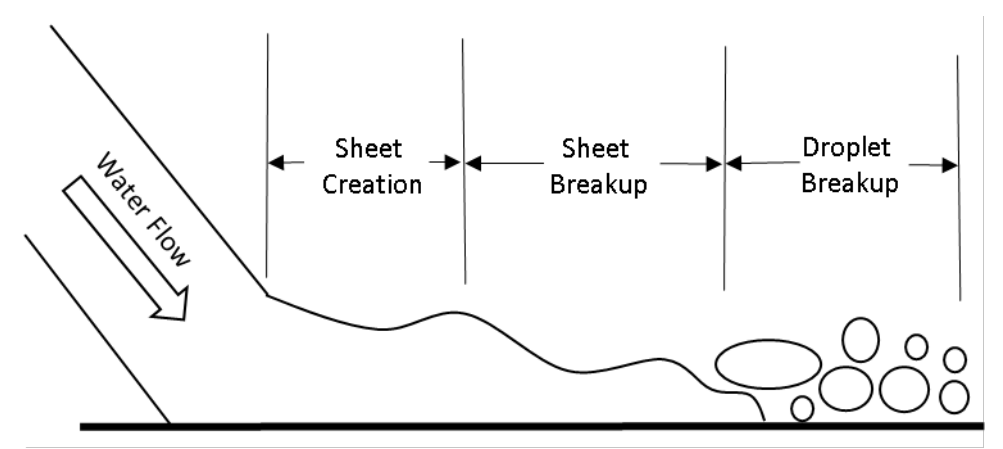

Fig. 5. Schematic view of a splash plate

In splash plates, the droplet velocity at the end of the plate depends on the initial velocity of the

water impact on the plate. In addition there is a dependence between the velocity and size of the 
252 splash plates; it is the physical mechanism of liquid breakup and atomization. The fundamental physics of

253 liquid breakup maintains that the free surface liquid with a high velocity must break up into smaller sizes.

254 The theory of atomization states that low-velocity liquids can retain their unity, but the other high-velocity

255 parts are divided and resized to satisfy the balance of inertia and surface tension forces. The Weber

256 number is the common parameter for the breakup process and it can explain this inverse dependence

257 easily (Sazhin, 2014).

258 Sarchami et al. (2010) showed that for a splash plate, the droplet velocity and size have an inverse 259 relationship. The research demonstrated that increasing the initial velocity from $10 \mathrm{~m} / \mathrm{s}$ to $30 \mathrm{~m} / \mathrm{s}$ creates 260 droplets of about $350 \mu \mathrm{m}$ to $250 \mu \mathrm{m}$ in diameter, respectively; therefore, increasing velocity produces 261 finer droplets and consequently the high-velocity droplets are small in size and vice versa.

There have been several studies on the size and velocity of droplets for sheet and droplet breakup.

263 These studies indicate that this relation is inverse. According to the breakup situation and associated 264 parameters, and considering the dominant mechanism of breakup, this inverse relation can vary. Adams et al. (1977) suggested a correlation for droplet sizes of a sheet breakup process as follows:

$$
D \times V^{0.55}=\text { constant }
$$

267 where $D$ and $V$ are the droplet diameter and velocity respectively. The constant depends on the viscosity, 268 surface tension, and density of the sheet, and also on dimensional characteristics which are fixed for a 269 breakup process. In this relation, droplet diameters are inversely proportional to the 0.55 th power of 270 droplet velocities.

271 In Ingebo's work (1984), which is experimental, an empirical correlation for droplet size and

272 Reynolds number is resented. The correlation is as follows:

$$
D \times V=\text { constant }
$$

274 The constant depends on the viscosity and density of the sheet and also the geometry of the plate. This 275 paper proposes another inverse dependence between the sizes and velocities of the droplets. 
277 breakup, which is a type of breakup in which droplets are split up after formation of a water bag, and

278 stripping breakup, which is splitting droplets by the contribution of surface shear force between air and

279 water, can be two dominant mechanisms in this stage. The Weber number is a useful criterion for 280 assessment of the breakup situation. According to Sazhin (2014), the Weber number, which is the criterion 281 for bag breakup, can be shown as follows:

$$
W e=\frac{\rho V^{2} D}{\sigma}
$$

where $\rho$ is air density, $\sigma$ is surface tension, and $W e$ is the Weber number. In the breakup condition, the surface tension and air density are considered constant. The breakup process is stopped when the Weber number reaches lower than a critical amount. Therefore, the Weber number for the end of the breakup process is considered constant and consequently, a new relation can be developed:

$$
D \times V^{2}=\text { constant }
$$

The constant depends on the surface tension, density and the Weber number. This inverse dependence occurs by a power of two. this type of breakup is suggested in terms of $W e / \sqrt{R e}$. or after manipulation,

$$
D \times V^{1.5}=\text { constant }
$$

The constant depends on the surface tension, viscosity, density and the Weber number. In this relation, 294 droplet diameters are inversely proportional to the 1.5th power of the droplet velocities.

297 of the droplets at the end of the breakup process, and the quality of this inverse proportion depends on the breakup conditions. Equations $(1,2)$ and $(4,5)$, which are the samples of size-velocity relations, 
confirm this overall inverse dependence. Other past research on the breakup process confirms this fact as well (Sazhin, 2014).

The above equations emphasise that at the end of the water breakup process, the bigger droplets have a lower velocity in comparison to the smaller droplets. The magnitude of velocity and size and the power of that inverse proportion depends on the details of the breakup mechanism. This observation is a significant fact that can be used for reaching a distribution of size and velocity of droplets in front of a vessel's bow. The power of that proportion varies around unity and the exact overall power cannot be found easily. The inverse proportion, however, is more important than the exact amount of the power. In this paper, we will assume that the overall breakup phenomenon is an average of those phenomena, and based on this assumption, the sizes and velocities are obtained and used.

\section{Droplet Trajectory Analysis}

311 Spray droplets are expected to move upward next to the bow and be injected into the free stream above

312 the bow tip in front of the vessel. The droplets are injected with various velocities and masses. Their kinetic 313 energies are different, and some of the droplets with a higher level of kinetic energy can travel in front of 314 the vessel, while others do not have enough kinetic energy to overcome gravity acceleration and will 315 quickly return to the sea. Very big and low-velocity droplets will return to the sea and the rest will meet 316 the free stream. means they enter the air stream in the opposite direction of the force of the gravity and also in the opposite direction of the wind velocity. Droplets are decelerated by the air stream and gravity and consequently change their direction to the wind direction and downward direction. The trajectory of the droplets in front of the vessel is modeled by the one-way modeling method and solved by a numerical 
322

323

324

325

326

327

328

329

330

331

332

334

335

method. By assuming a uniform flow in the direction opposite that of the vessel, and knowing the droplet size and velocity, solving the spray trajectory becomes possible.

Spherical droplets, having a density of $\rho_{d}$ and a diameter of $D_{d}$, which is very small compared to the flow length scale (the bow dimension), are assumed. Applying Newton's Second Law for the droplet motion and substituting the body force, drag force, and added mass force, will result in the governing equation of the droplet trajectory. The governing equation related to droplet movement and the forces acting on them can be derived as follows (Dehghani et al. 2013):

$$
\begin{aligned}
m_{d} \frac{d \boldsymbol{V}_{\boldsymbol{d}}}{d t}= & \rho_{d} \forall_{d} \mathbf{g}-C_{d r} \frac{\pi D_{d}^{2}}{8} \rho_{a}\left|\boldsymbol{V}_{\boldsymbol{d}}-\boldsymbol{V}_{\boldsymbol{a}}\right|\left(\boldsymbol{V}_{\boldsymbol{d}}-\boldsymbol{V}_{\boldsymbol{a}}\right) \\
& +\rho_{a} \forall_{d} C_{a d} \frac{D\left(\boldsymbol{V}_{\boldsymbol{a}}-\boldsymbol{V}_{\boldsymbol{d}}\right)}{D t}+\rho_{a} \forall_{d}\left(\frac{D \boldsymbol{V}_{\boldsymbol{a}}}{D t}-\mathbf{g}\right)
\end{aligned}
$$

where $m_{d}$ is mass of droplet, $t$ is time, $\boldsymbol{V}_{\boldsymbol{d}}$ is droplet velocity, $\boldsymbol{V}_{\boldsymbol{a}}$ is air velocity, $\rho_{d}$ is water density, $\forall_{d}$ is droplet volume, $\mathbf{g}$ is gravity, $C_{d r}$ is drag coefficient, $D_{d}$ is droplet diameter, $\rho_{a}$ is air density, and $C_{a d}$ is added mass force coefficient. The coefficient $C_{a d}$ is assumed 0.5 and $C_{d r}$ can be calculated as follows (Dehghani et al. 2009):

$$
C_{d r}=\left\{\begin{array}{lr}
\frac{24}{R e} & R e<1 \\
\frac{24}{R e}\left(1+0.15 R e^{0.687}\right) & 1<R e<1000 \\
0.44 & R e>1000
\end{array}\right.
$$

In order to solve this equation, its unknowns are calculated separately. Substituting the unknowns leads to a set of ordinary differential equations.

$$
\begin{gathered}
\dot{x}=\frac{d x}{d t}, \quad \ddot{x}=\frac{d^{2} x}{d t^{2}}, \quad \ddot{x}=-\frac{3 C_{d r}}{4 D\left(\gamma+C_{a d}\right)}(\dot{x}-U) \sqrt{(\dot{x}-U)^{2}+\dot{z}^{2}} \\
\dot{z}=\frac{d z}{d t}, \quad \ddot{z}=\frac{d^{2} z}{d t^{2}}, \quad \ddot{z}=\left(\frac{1-\gamma}{\gamma+C_{a d}}\right) \mathrm{g}-\frac{3 C_{d r}}{4 D\left(\gamma+C_{a d}\right)}(\dot{z}) \sqrt{(\dot{x}-U)^{2}+\dot{z}^{2}}
\end{gathered}
$$

where $x, \dot{x}, \ddot{x}, z, \dot{z}$, and $\ddot{z}$, are position, velocity, and acceleration of the droplets, $\gamma$ is liquid density to air density ratio, and $U$ is the relative velocity of wind to vessel. The initial conditions are droplet sizes and velocities. This set of six equations and six unknowns can be solved by a numerical scheme. 
344 of the droplets, but in reality the mass of vaporized water is negligible in comparison to the original mass

345 of the droplets. Therefore, the small mass reduction due to the vaporization is ignored. The numerical

346 solution is limited to modeling of the droplet trajectory just in front of the vessel. Droplets traveling on

347 the deck are dependent on the vessel geometry and obstacles on the deck, which is beyond the scope of 348 this paper.

The input data for the droplet trajectory model are wind velocity, injection angle, droplet sizes and droplet velocities. The present model simulates the wave-impact sea spray that MFV on the Sea of Japan experienced (Borisenkov et al., 1975), and therefore in this work the situation of that vessel and the 352 atmospheric conditions of that time will be used in the analysis. The wind velocity is considered to be the same as the MFV on the Sea of Japan. The range of droplet sizes is chosen by using the work of Ryerson 354 (1995) which is about 0-7000 $\mu \mathrm{m}$. The initial velocities must be chosen to create a spray height recorded 355 in the same as the report of the MFV of the Sea of Japan (Borisenkov et al., 1975). By having a specific amount of sea water that creates the cloud of spray and also having a distribution of size and velocity of

357 the droplets, the concentrations of droplets are determined. The injection angle will be assumed as the 358 overall injection angles of droplets after the sheet-water breakup. In a steady state situation, this angle can be considered equal to the bow angle. The real phenomenon is transient, and the hitting angle of the wave and bow occur such that the oscillation of the vessel will result in different injection angles. 361 Therefore the injection angle can be assumed about the bow angle and by considering a reasonable 362 tolerance, the variation of that angle can be addressed.

By using a range of velocities and sizes for droplets and considering the inverse relations for sizes 364 and velocities, a set of curves can be plotted. Figure 6 shows the distribution of the size and velocity of 365 droplets for a droplet range of 0-7000 $\mu \mathrm{m}$ and a velocity range of $0-40 \mathrm{~m} / \mathrm{s}$. The bottom-right corner of 
367 velocities, the drag force, which is a contraflow force, slows them down rapidly. The length scale of the

368 bow is bigger than the length scale of the splash plate and thus the effect of drag force in reducing the

369 velocity of high-velocity droplets will be more considerable than the splash plates. In reality, the high-

370 velocity and small droplets will be affected by this drag force and as a result it reduces their velocities at

371 a short distance from the creation area. Therefore, this is a reasonable assumption to correct the high-

372 velocity region and limit it to the maximum velocity. This correction shifts the right-hand half of the curves

373 of Fig. 6 a little lower. In moving more towards the higher velocity region, this shift will be more

374 considerable.

375

376

377

378

379

380

381

382

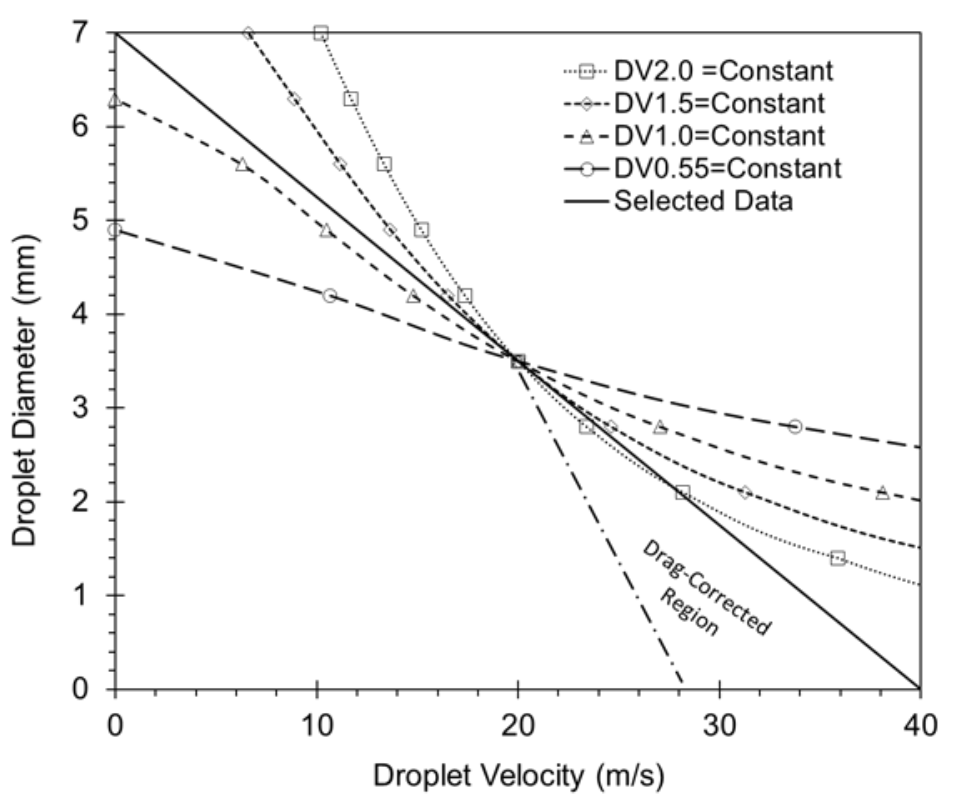

Fig. 6. Sample of size-velocity dependence for droplets

The breakup phenomenon can have combinations of some breakup mechanisms that have been explained. Figure 6 shows the effects of those mechanisms on the size and velocity of the droplets. It is difficult to determine that just one of those mechanisms is effective in determining the sizes and velocities. All of them can contribute to forming the cloud of spray. It is reasonable to assume approximate 
overall results for those outputs. A simple inverse size-velocity dependence which is chosen in this study and named as selected data is the linearly decreasing line in Fig. 6. This inverse dependence curve covers the weakness of the other models in the high-velocity region. It also reflects the overall effects of the other models in the low-velocity and big-sized regions.

\section{Numerical Results}

In a wave-impact spray event, size and velocity distributions of the droplets are essential for determining the exact rate of water delivery to every part of a vessel. Previous research has shown that the droplet size and velocity at the end of the breakup process have an inverse dependence. This means that higher velocity droplets are smaller than lower velocity droplets. The exact droplet size and velocity depends on the wave specification, vessel velocity, wind velocity, and the quality of the impact. By knowing the range of velocities and sizes of the droplets at the highest level of the bow, and considering the velocity direction of droplets nearly parallel with the bow, a wind velocity of $11 \mathrm{~m} / \mathrm{s}$, and a vessel velocity of $2.83 \mathrm{~m} / \mathrm{s}$, the same as the situation of the MFV of the Sea of Japan which is reported by Borisenkov et al. (1975), the

397 effect of these variables on the spray cloud and LWC can be examined. Table 1 shows the values for the 398 droplet trajectory.

Table 1. Working conditions of droplet trajectory in front of the vessel

\begin{tabular}{lcc}
\hline Parameters & Reference & Unit \\
& Values & \\
\hline Initial Velocity of Droplets & $0-40$ & $\mathrm{~m} / \mathrm{s}$ \\
Droplets Diameter & $0-7000$ & $\mu m$ \\
Injection Angle & 20 & Degree \\
\hline
\end{tabular}


Figure 7 shows a schematic view of the bow, injection location and angle of droplets, wind velocity, and vessel velocity. The coordinate system is attached to the vessel and the origin is on the highest point of the bow in front of the vessel. As mentioned, this study is limited to the survey of spray creation in front of the vessel. The droplet trajectory on the vessel is beyond the scope of this research. The space of 406 interest is confined between the positive coordinate axes of $x$ and $z$.

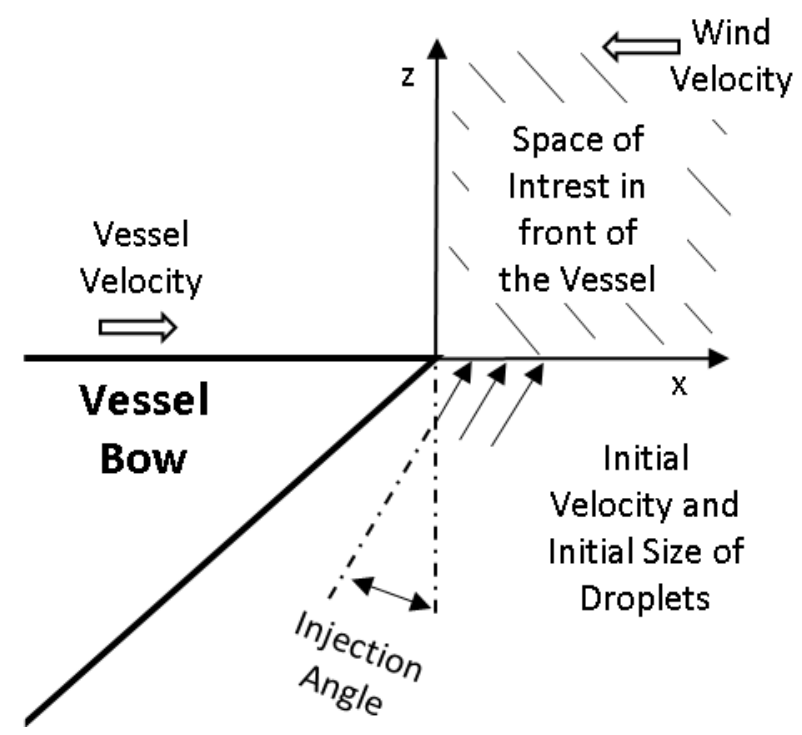

Fig. 7. Schematic view of the bow and the variables

The first output of the droplet trajectory will be the dispersion of a spray cloud in front of the vessel.

412 Figure 8 shows the development of a spray cloud over time. The spray cloud will cover the entire front of 413 a vessel in less than $0.48 \mathrm{~s}$. At this point, the spray is not at its maximum height over the vessel. The spray 414 cloud can further extend upwards on the vessel and the geometry of the deck can affect it. The maximum counter-direction horizontal movement of the spray cloud in front of the vessel is 416 about $0.45 \mathrm{~m}$. The spray cloud is extended vertically very rapidly. After $0.16 \mathrm{~s}$, the height of the spray 417 reaches about $1.7 \mathrm{~m}$ and, at $0.24 \mathrm{~s}$, it reaches a height of $2.7 \mathrm{~m}$. At $0.32 \mathrm{~s}$, the spray has moved vertically 418 just $0.8 \mathrm{~m}$, to a height of $3.5 \mathrm{~m}$. At a time of $0.4 \mathrm{~s}$, the spray cloud is extended completely in the vertical 

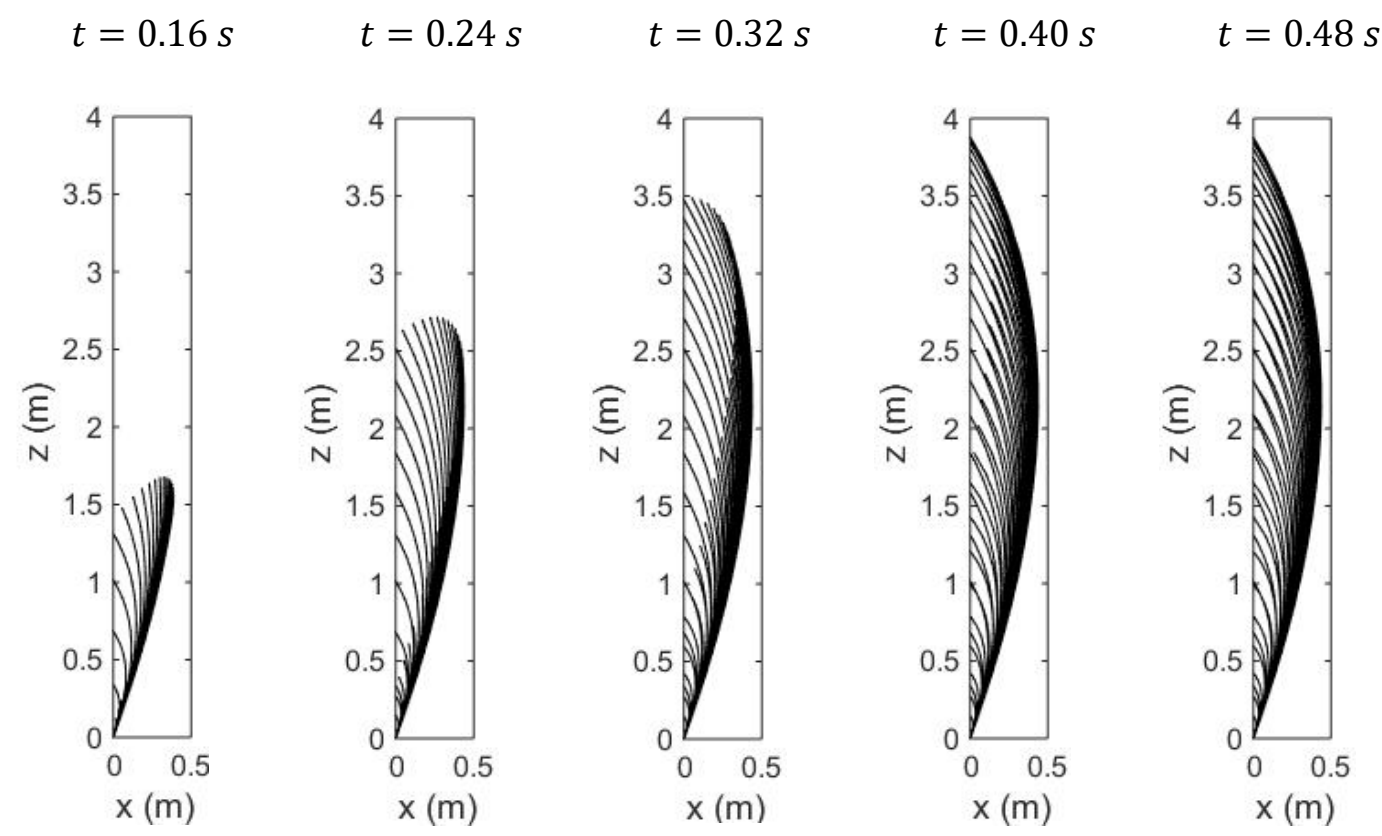

424

425

426

427

428

429

430

431

where $z$ is the elevation above the deck of MFE (Zakrzewski, 1987) and $w$ is the LWC.

Fig. 8. Spray cloud development in front of a vessel. Vertical development versus horizontal development in front of a vessel (Compatible with the coordinate axis of Fig. 7) numerical solution. Figure 9 shows a comparison between the numerical results and the LWC reported by Borisenkov et al. (1975). The proposed relation that represents the liquid water content is given by

$$
w=24.2 \times \exp (-0.55 z) \quad g r_{\text {water }} / m^{3} \text { air }
$$

The liquid water content from the field observations can be used as a reference to check the 
441 integration intervals and points. Due to the integration on the discrete phase of water droplets, the obtained LWCs may not be smooth curves. The quality of the numerical curves depends on the number

444 inhomogeneous distribution of droplets and also the integration intervals and points. Figure 9 shows that

445 increasing the integration points can refine these regions and also reduce the number of resultant data 446 on the curves. The numerical results are aligned with the field observations. They almost match the exponential 448 behaviour of the experimental results. For the higher altitudes, they are well matched and for lower

Fig. 9. Comparison of liquid water content of the numerical approach and a fit to the field observations

by Borisenkov et al. (1975)

cloud. The numerical results are obtained based on integration of the properties of this inhomogeneous discrete phase of water droplets in the vertical direction. This integration requires the definition of space intervals and points on the relevant domain. Figure 9 shows the numerical results in different cases of of intervals and points on the subdivided ranges. Some points on the curves are the result of the

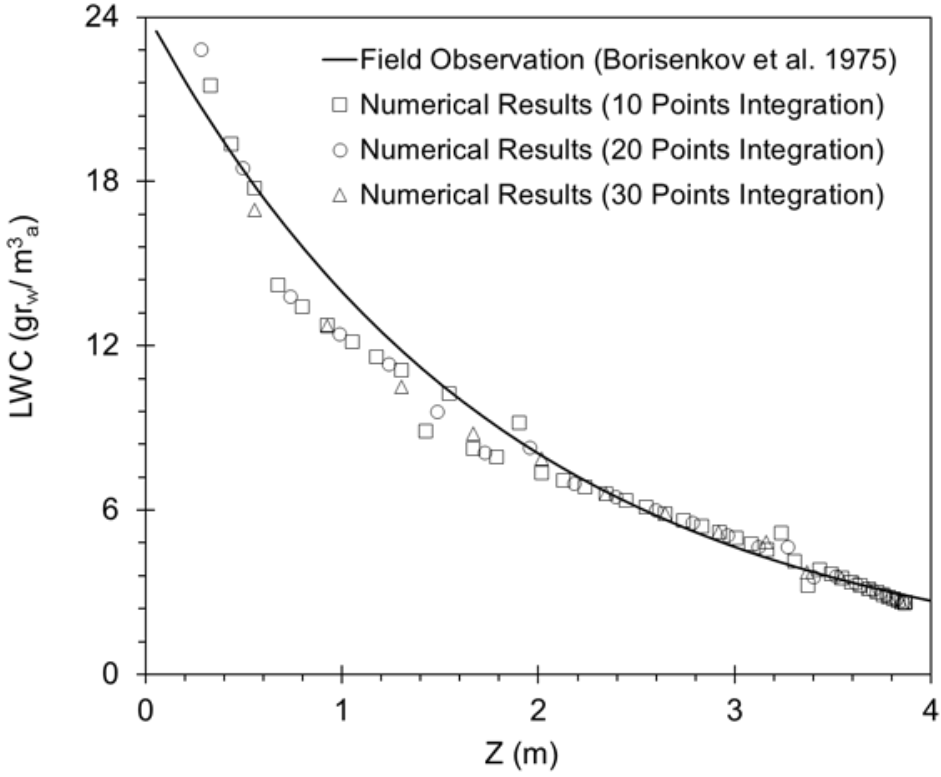


altitudes, some small differences exist. The main result of this comparison emphasizes that the

450 distribution of sizes, which is inversely proportional to the distribution of velocities, is compatible with the

451 exponential behaviour of vertical distribution of the LWC. In the next section, it will be shown that the

452 other relations between the sizes and velocities cannot yield an exponential function and the most valid

453 relation is an inversely proportional relation.

454

455 5. Sensitivity Analysis

456 A sensitivity analysis was conducted to determine how sensitive this model is to changes in the value of

457 the input parameters. By showing how the model behavior responds to changes in input parameter

458 values, the sensitivity analysis is a useful tool in model building as well as in model evaluation. The first

459 test will assess the sensitivity of the model to various size-velocity dependencies of the droplets. Referring

460 to past studies (Lozowski et al., 2000; Zakrzewski, 1987; Shipilova et al., 2012; Horjen, 2013; Kulyakhtin

461 and Tsarau, 2014) and assumptions for choosing the initial velocity and size in the spray cloud, it is possible

462 to categorize the assumptions and other possible cases to some individual categories. There are four

463 individual cases for the dependence of velocity on the size of droplets. The first case is an inverse

464 dependence, which has high-velocity, small-sized droplets and low-velocity, large-sized droplets. The

465 second case is the opposite of the first case. This case, which will be named the co-direction dependence,

466 considers high-velocity, large-sized droplets and low-velocity, small-sized droplets. The third case is a

467 constant velocity and arbitrary droplet size, and the fourth case is a constant droplet size and arbitrary

468 droplet velocity. It will be shown that, except in the first case, which has been chosen for this study, the

469 other cases do not yield an acceptable LWC curve.

470 Figure 10 shows the response of the model to various inputs of size-velocity dependence. The co-

471 direction case is the least appropriate case and predicts a nearly linear curve with an opposite slope with

472 respect to the field observation data. The other case occurs when velocity is constant, which predicts a 
474 fit to an exponential correlation of the field observation. The curve is approximately horizontal, and 475 therefore it is not a suitable case to be chosen.

476

477

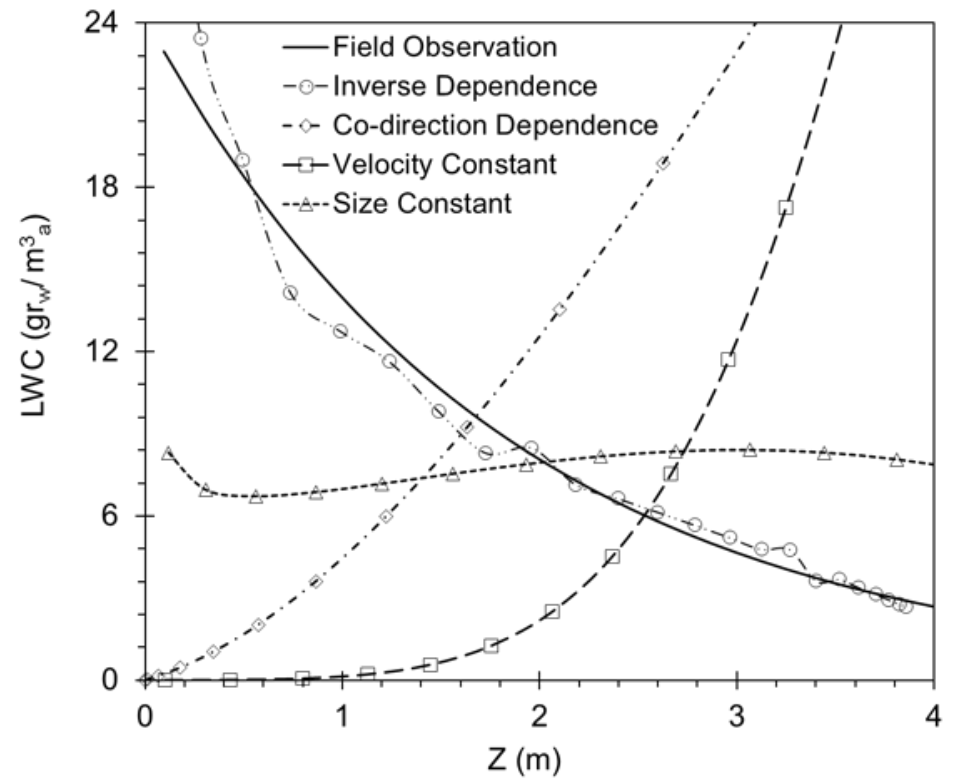

Fig. 10. Sensitivity of the model to various size-velocity dependences

As shown in Fig. 10, the assumption of a constant velocity and size cannot adequately yield the exponential form of the LWC formula. The co-direction dependence is another inappropriate assumption for droplet size and velocity information. This analysis shows that the previous assumptions are not suitable. The other significant conclusion of this analysis is the importance of determining the distribution of size and velocity instead of assuming a constant velocity and size. The only acceptable case is an inverse velocity-size dependence. This case can be fit to an exponential curve and is in alignment with the field observation reports. The sensitivity analysis of size-velocity dependence shows that the model is highly sensitive to the size-velocity dependence. This means the only suitable case of size-velocity dependence 
in wave-impact sea spray is inverse dependence. This sensitivity analysis is good evidence for the necessity

489 of the inverse size-velocity dependence in the wave-impact breakup.

The other parameters that test the sensitivity of the model are the maximum velocity of droplets,

maximum size of droplets, and injection angle. The various degrees of these parameters are shown in

492 Table 2. In each case of sensitivity analysis the response of the model is evaluated by varying a parameter

493 in the mentioned range of Table 2. The other parameters are kept at the reference values.

494

Table 2. Various amounts of input data for sensitivity analysis

\begin{tabular}{|c|c|c|c|c|c|c|}
\hline \multirow{2}{*}{ Parameters } & \multirow{2}{*}{ Unit } & \multicolumn{5}{|c|}{ Range of Values for Sensitivity Analysis } \\
\hline & & & & Reference & & \\
\hline Initial Velocity of Droplets & $\mathrm{m} / \mathrm{s}$ & $0-20$ & $0-30$ & $0-40$ & $0-50$ & $0-60$ \\
\hline Droplet Size (Diameter) & $\mu m$ & $0-5000$ & $0-6000$ & $0-7000$ & $0-8000$ & $0-9000$ \\
\hline Injection Angle & Degree & 10 & 15 & 20 & 25 & 30 \\
\hline
\end{tabular}

Figure 11 shows the response of the model to various initial velocities. This test is conducted by using the reference values of droplet size and injection angle. The model is very sensitive to the variation of droplet velocities. The most considerable response of the system to a variation of the maximum initial

500 velocity of droplets appears in the higher altitudes. In the case of lower velocities, droplets create a short 501 cloud spray and therefore the LWC is zero for the high altitude. The model is more sensitive to lower 502 velocities than to higher ones. At high velocities, the maximum deviation is less than 8 percent but for low 503 velocities the deviation is more than 50 percent. For all cases, the LWC for the heights of less than $1 \mathrm{~m}$ are 504 very close to each other, but by increasing the height, the lower velocity cases start to diverge. 


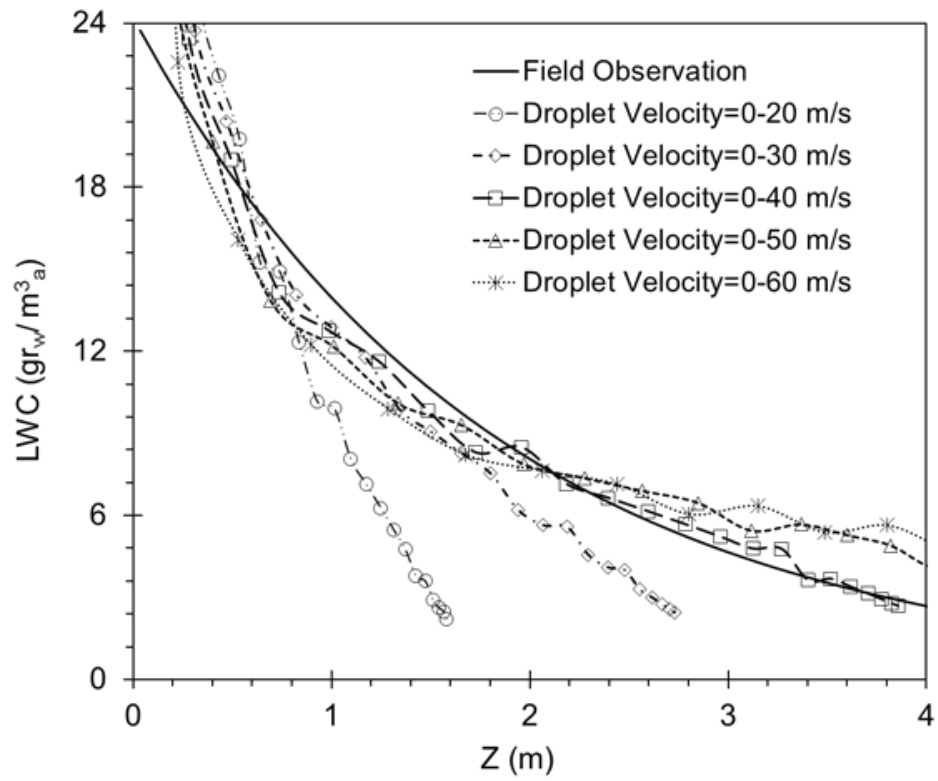

Fig. 11. Sensitivity response of model to various initial velocities

510 the response of the model to this parameter. Increasing the droplet size results in an increase of LWC. The

511 system is more sensitive to larger droplets than to smaller droplets. The volume of water is proportional

512 to the third power of the droplet diameter and it can be the reason for the high sensitivity of the model

513 to bigger droplets. When decreasing the maximum size of the droplets, they cannot travel as high as the

514 reference value case because of the drag force. The drag force affects the small droplets more easily, and

515 they will be in the wind direction after a short time. Therefore they cannot reach the highest altitude. 


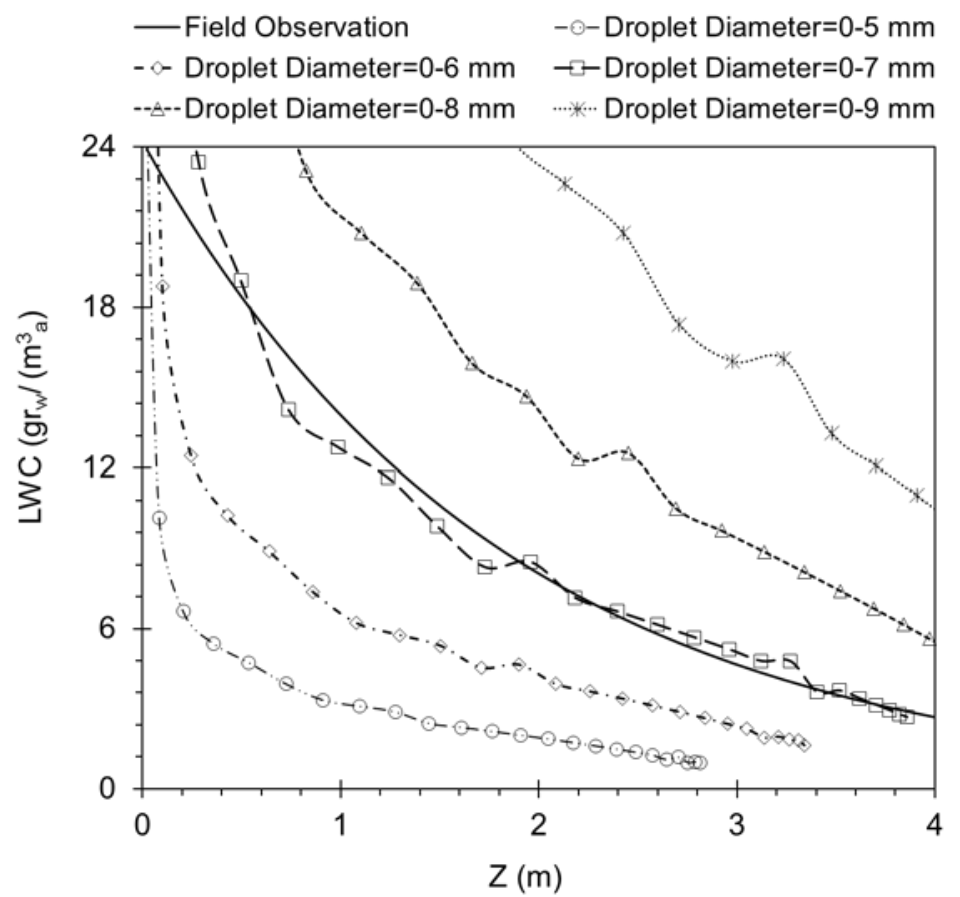

Fig. 12. Sensitivity response of model to droplet sizes

520 shows the response of the model to this parameter. The model is more sensitive to smaller injection

521 angles than to greater angles. In comparison to the higher injection angles, the smaller injection angles

522 create lower values of horizontal components and higher values of vertical components of the initial

523 velocity of droplets. The greater injection angles result in lower values of LWC in comparison to that of 524 smaller injection angles. In addition, due to smaller horizontal components of the initial velocity of 525 droplets, the droplets take a shorter path and consequently arrive on the deck before reaching the higher 526 altitudes. Therefore the LWC of heights greater than $2.6 \mathrm{~m}$ for the injection angles of 10 degrees is zero.

527 This means that decreasing the injection angle decreases the effective height of the LWC. The greater 528 injection angles yield higher values for the LWC in comparison to the reference value. 


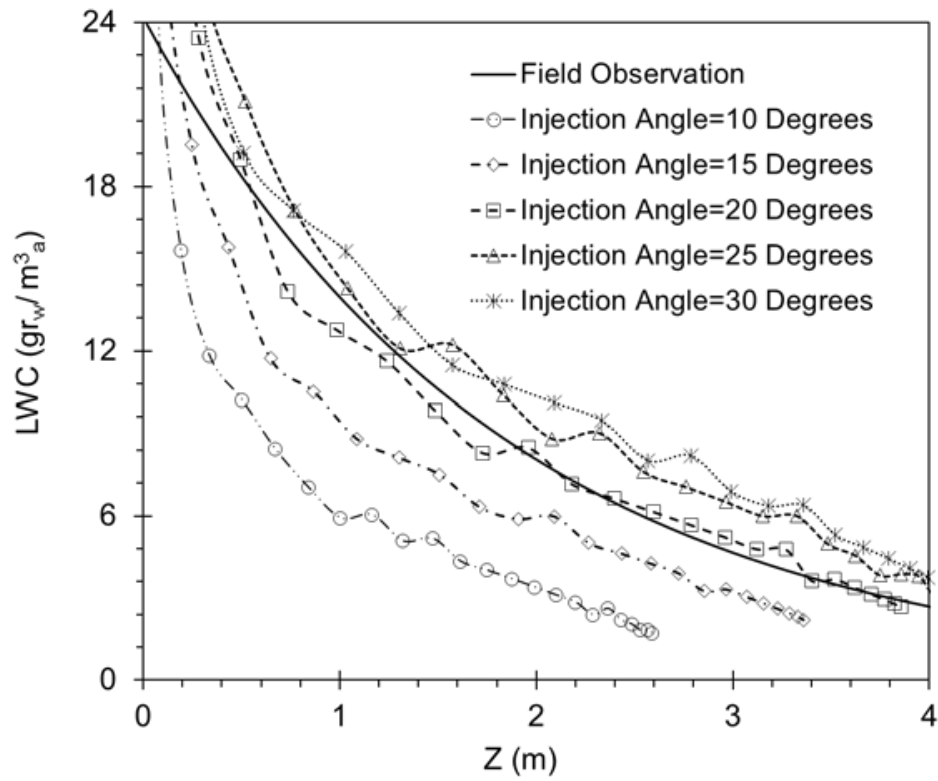

Fig. 13. Sensitivity analysis of model to various injection angles

534 to droplet size or droplet velocity. The model is very sensitive to droplet sizes and the LWC is affected by 535 changing the sizes strongly. The velocity range is the case that can affect the effective height of the LWC. 536 Increasing the maximum velocity increases the height of the LWC and vice versa.

\section{6. Conclusions}

539 A new model for the prediction of the characteristics of wave-impact sea spray in front of a vessel was 540 developed. The sheet breakup and droplet breakup in front of the vessel bow showed that there is a 541 significant dependence between the sizes and velocities of resulting droplets after breakup. High-velocity 542 droplets tend to split into fine droplets and low-velocity droplets tend to keep their size. This makes a 543 size-velocity connection in the droplets which are injected into the atmosphere in front of a vessel. At the 544 injection points, next to the highest points of the bow, the bigger droplets have lower velocities and vice 545 versa. By using the droplet trajectory and considering the vessel velocity and wind velocity, the spray cloud 
546 in front of the vessel can be predicted. The drag force, added mass force, and body force are effective

547 forces in this analysis. The wave-impact sea spray is expanded in front of the vessel in $0.48 \mathrm{~s}$. The LWC is

548 calculated by considering the droplet arrangement, size, and velocity at the arrival section in front of the 549 vessel.

550 Sensitivity analyses show that the model is very sensitive to size-velocity dependence. Co-direction 551 dependence for the size and the velocity, independence of droplet size from velocity, and independence 552 of droplet velocity from size, will result in unacceptable values of the LWC. The inverse dependence 553 between size and velocity is the only dependence that can adequately predict the correct value of the 554 LWC. The sensitivity analysis shows that the system is very sensitive to small injection angles, big droplets, 555 and low-velocity droplets. The proposed inverse size-velocity dependence for the injected droplets in the 556 atmosphere in front of the vessel is a useful new model that can fill a knowledge gap about the size and

557 velocity of droplets due to the impact of a wave on a bow. This proposed model was verified by comparing 558 it to the LWC measured by Borisenkov et al. (1975). By using this new model, a more accurate calculation 559 of the water impact across a vessel can be achieved.

560

\section{Acknowledgments}

562 The authors gratefully acknowledge the financial support from Statoil (Norway), MITACS, and Petroleum 563 Research Newfoundland and Labrador (PRNL) for this research.

\section{References}

566 Adams, T.N., Frederick, W.J., Grace, T.M., Hupa, M., Lisa, K., Jones, A.K., Tran, H., 1977. Kraft Recovery 567 Boilers. TAPPI Press. 
Ahmed, M., Ashgriz, N., Tran, H.N., 2009. Break-Up Length and Spreading Angle of Liquid Sheets Formed by Splash Plate Nozzles. Journal of Fluid Engineering, 131, 1-9.

570

Ashgriz N., Washburn R., Barbat T., 1996. Segregation of drop size and velocity in jet impinging splash-

571 plate atomizers. International Journal of Heat and Fluid Flow, 17 (5), 509-516.

572 Borisenkov, Y.P., Zablockiy, G.A., Makshtas, A.P., Migulin, A.I., Panov, V.V., 1975. On the approximation of 573 the spray cloud dimensions. Arkticheskii I Antarkticheskii Nauchno-Issledovatelskii Instit. Trudy 317. 574 Leningrad: Gidrometeoizda (in Russia), pp. 121-126.

575 Bussmann, M., Chandra S., Mostaghimi J., 2000. Modeling the splash of a droplet impacting a solid surface. 576 Phys. Fluids 12, 3121.

577 Dehghani, S.R., Saidi, M.H., Mozafari, A.A., Ghafourian, A., 2009. Particle Trajectory in a Bidirectional 578 Vortex Flow. Particulate Science and Technology, 27, 16-34.

579 Dehghani, S.R., Saidi, M.H., Mozafari, A.A., Soleimani, F., 2013. Particle Dispersion Dependency on the 580 Entrance Position in Bidirectional Flow. Particulate Science and Technology, 31, 576-584.

581 Forest, T.W., Lozowski, E.P., Gagnon, R., 2005. Estimating Marine Icing on Offshore Structures using 582 RIGICE04. IWAIS XI, Montréal, June 2005.

583 Horjen, I., 2013 Numerical modeling of two-dimensional sea spray icing on vessel-mounted cylinders, Cold 584 Regions Science and Technology, 93, 20-35.

585 Horjen, I., Carstens, T., 1989. Numerical modelling of the sea spray icing on vessels. In Proc. 10th Int. POAC 586 Conf., Lulea, Sweden, vol. 3, pp. 694-704.

587 Greco, M., Bouscasse, B., Lugni, C., 2012. 3-D seakeeping analysis with water on deck and slamming. Part 588 2: Experiments and physical investigation. Journal of Fluids and Structures, 33, 148-179.

589 Greco, M., Lugni, C., 2012. 3-D seakeeping analysis with water on deck and slamming. Part 1: Numerical 590 Solver. Journal of Fluids and Structures, 33, 127-147. 
591 Gu, H.B., Qiana, L., Causona, D.M., Minghama, C.G., LinbNumerical P., 2014. Simulation of water impact 592 of solid bodies with vertical and oblique entries. Ocean Engineering. 75, 128-137.

593 Ingebo, D., 1984. Atomization of liquid sheets in high pressure air flow. NASA-Lewis Research Center, 594 Cleveland, $\mathrm{OH}$.

595 Kaspenberg, G.K., 2011. Slamming of ships: where are we now? Phil. Trans. R. Soc. A, 369, 2892-2919.

596 Kulyakhtin, A., Tsarau, A., 2014. A time-dependent model of marine icing with application of 597 computational fluid dynamics. Cold Regions Science and Technology, 104-105, 33-44.

598 Lozano. A., García-Olivares, A., Dopazo, C., 1998. The instability growth leading to a liquid sheet breakup. $59910(9), 2188-2197$.

600 Lozowski, E.P., Szilder, K., Makkonen, L., 2000. Computer simulation of marine ice accretion. Royal Society, $601358,2811-2845$.

602 Makkonen, L., 1984. Atmospheric icing on sea structures. US Army Corps of Engineers, Cold Regions 603 Research \& Engineering Laboratory, CRREL MONOGRAPH 84.2.

604 Ryerson, C.C., 1995. Superstructure spray and ice accretion on a large U.S. Coast Guard cutter. 605 Atmospheric Research, 36 (3-4), 321-337.

606 Ryerson, C.C., 2011. Ice protection of offshore platforms. Cold Regions Science and Technology, 65 (1), $60797-110$

608 Sarchami, A., Ashgriz, N., Tran, H., 2010. An atomization model for splash plate nozzles. AIChE Journal, 56 $609 \quad(4), 849-857$.

610 Sazhin, S., 2014. Droplet and sprays. Springer-Verlag London.

611 Shipilova, O., Kulyakhtin, A., Tsarau, A., Libby, B., Moslet, P.O., Loset, S., 2012. Mechanism and Dynamics 612 of Marine Ice Accretion on Vessel Archetypes, OTC-23762-MS, OTC Arctic Technology Conference, 3-5 613 December, Houston, Texas, USA. 
614 Zakrzewski, W.P., 1987. Splashing a ship with collision-generated spray. Cold Regions Science and 615 Technology, $14(1), 65-83$.

616 Zakrzewski, W.P., Lozowski, E.P., 1988. Estimating the extent of the spraying zone on a sea-going ship.

617 Ocean Engineering. 15 (5), 413-429. 